\title{
Diisooctyl Sebacate-Containing Nickel Nanoparticles for Lubrication of Steel Sliding Parts under Magnetic Fields
}

Jiasheng Hü, Chaofeng Wang ${ }^{b}$, Pingyu Zhang ${ }^{a}$, Shengmao Zhang ${ }^{a}$, Yujuan Zhang ${ }^{a}$ * ${ }^{a}$ Engineering Research Center for Nanomaterials, Henan University, Kaifeng 475004, P. R. China

${ }^{b}$ Special equipment safety inspection and research institute of Henan Province, Zhengzhou, 450008, P. R. China

*Dr. Shengmao Zhang: Phone: (+86)371-25152066, Fax: (86) 371-25152066, Email: zsm@henu.edu.cn

*Dr. Yujuan Zhang: Phone: (+86)371-25152055. Fax: (86) 371-25152066, E-mail: 172186033@qq.com 


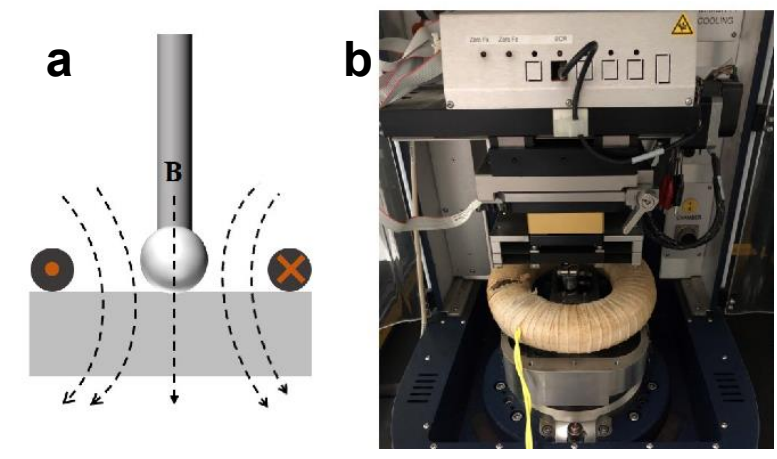

Figure S1. Schematic diagram of friction and wear test under magnetic field. 
Table S1 Friction and wear test condition.

\begin{tabular}{ll}
\hline Item & Value \\
\hline Frequency $(\mathrm{Hz})$ & $1,5,10$ \\
Load $(\mathrm{N})$ & $100,300,500$ \\
Magnetic field (mT) & $20,25,30$ \\
Sliding distance (mm) & 5 \\
Time (min) & 10 \\
\hline
\end{tabular}


Table S2 Details of the bearing steel sliding pair.

\begin{tabular}{lll}
\hline & \multicolumn{2}{l}{ Value } \\
\cline { 2 - 3 } Item & Upper specimen & Lower specimen \\
\hline Material & GCr 15 & AISI-1045 steel \\
Diameter $(\mathrm{mm})$ & 12.7 & $40 \times 30 \times 5$ \\
HRc & 62 & 60 \\
Elastic modulus $(\mathrm{GPa})$ & 205 & 200 \\
Poisson ratio & 0.3 & 0.28 \\
\hline
\end{tabular}



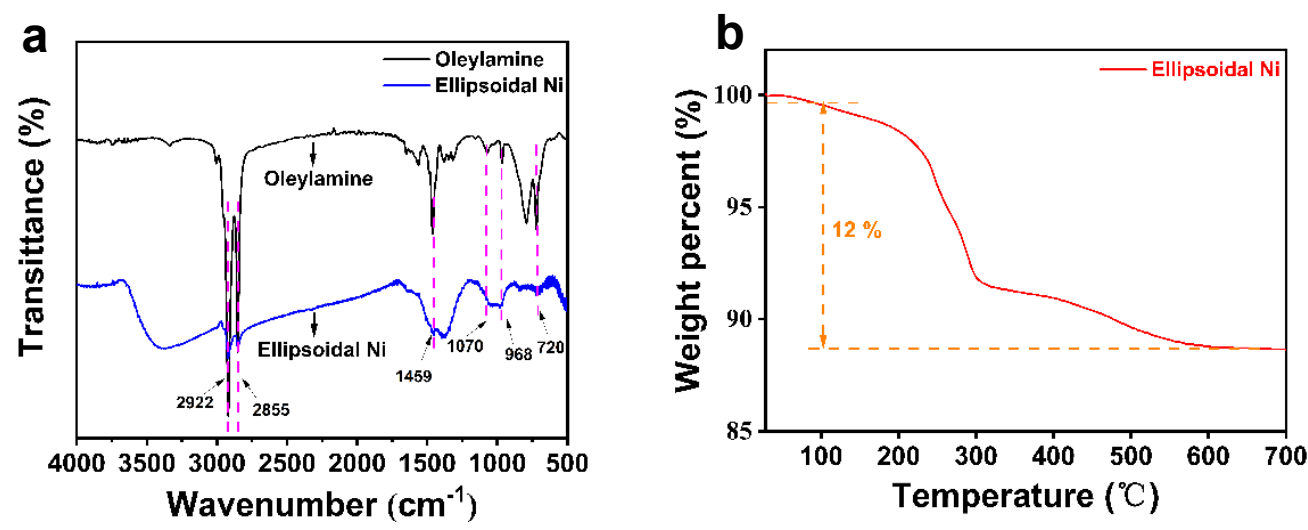

Figure S2. (a) FTIR spectra of oleylamine and ellipsoidal nickel nanoparticle, (b) Thermal weight loss curve of ellipsoidal Ni nanoparticle. 


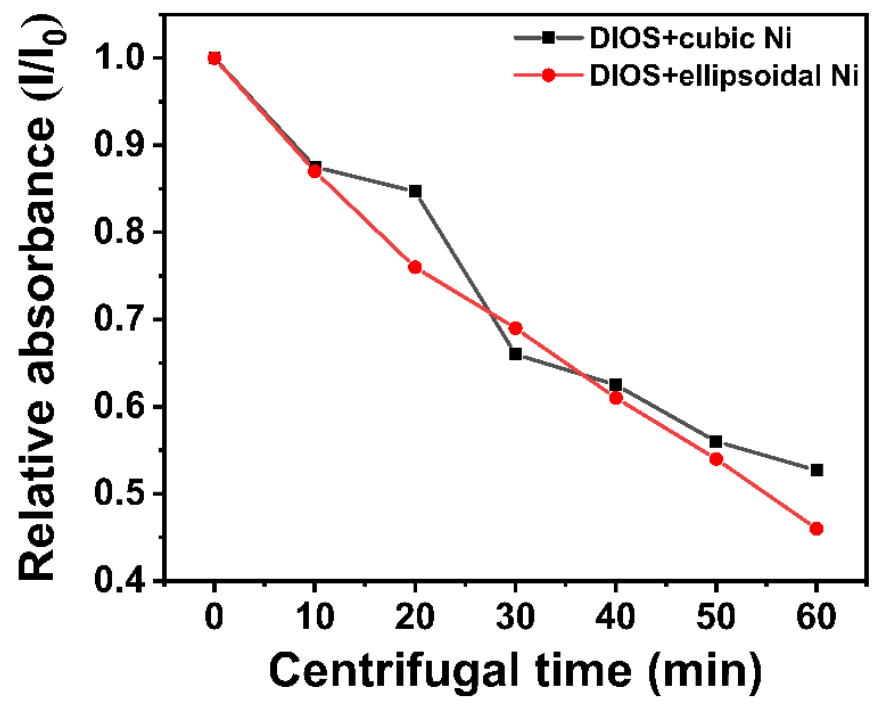

Figure S3. Relative light absorbance intensity of cubic and ellipsoidal Ni nanoparticles in DIOS after centrifugation for different duration. 
Table S3 Magnetic properties of two kinds of Ni nanoparticles.

\begin{tabular}{llll}
\hline Sample & $M_{\mathrm{s}}\left(\mathrm{emu} \cdot \mathrm{g}^{-1}\right)$ & $M_{\mathrm{r}}\left(\mathrm{emu} \cdot \mathrm{g}^{-1}\right)$ & $H \mathrm{c}(\mathrm{Oe})$ \\
\hline Cubic Ni & 46.3 & 2.48 & 26.6 \\
Ellipsoidal Ni & 47.6 & 0.78 & 9.6 \\
\hline
\end{tabular}



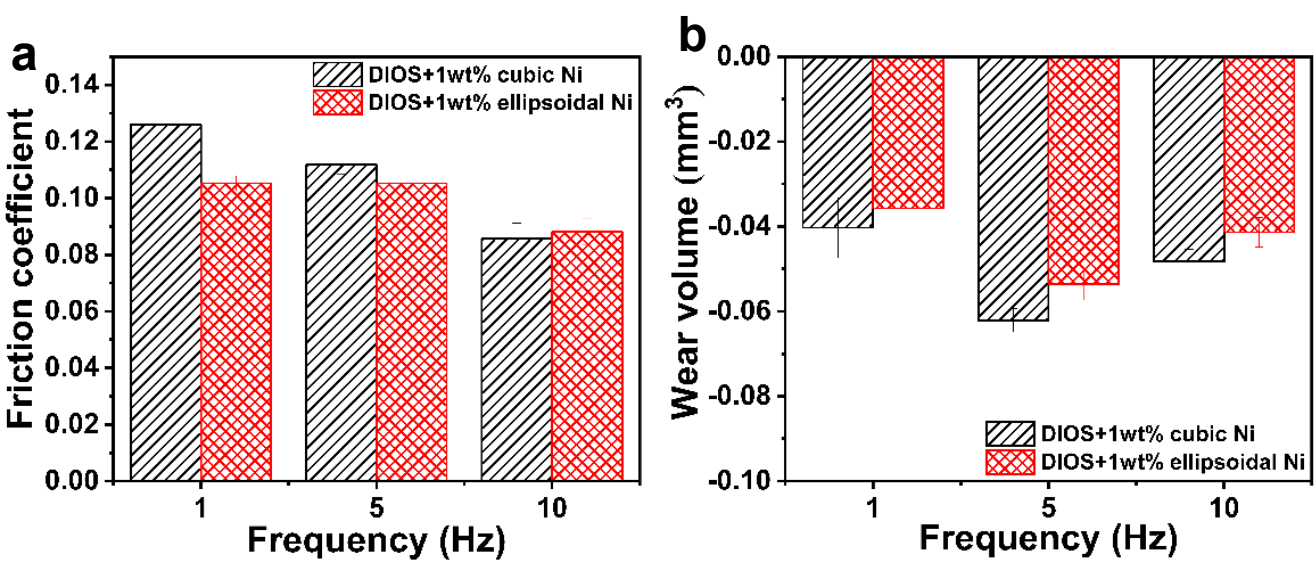

Figure S4. Friction coefficient (a) and wear volume (b) of lower steel block lubricated with DIOS containing 1\% Ni nanoparticles at different frequency under magnetic field (Magnetic field: $20 \mathrm{mT}$, load: $100 \mathrm{~N}$, additive concentration: $1 \%$, amplitude: $5 \mathrm{~mm}$ ). 

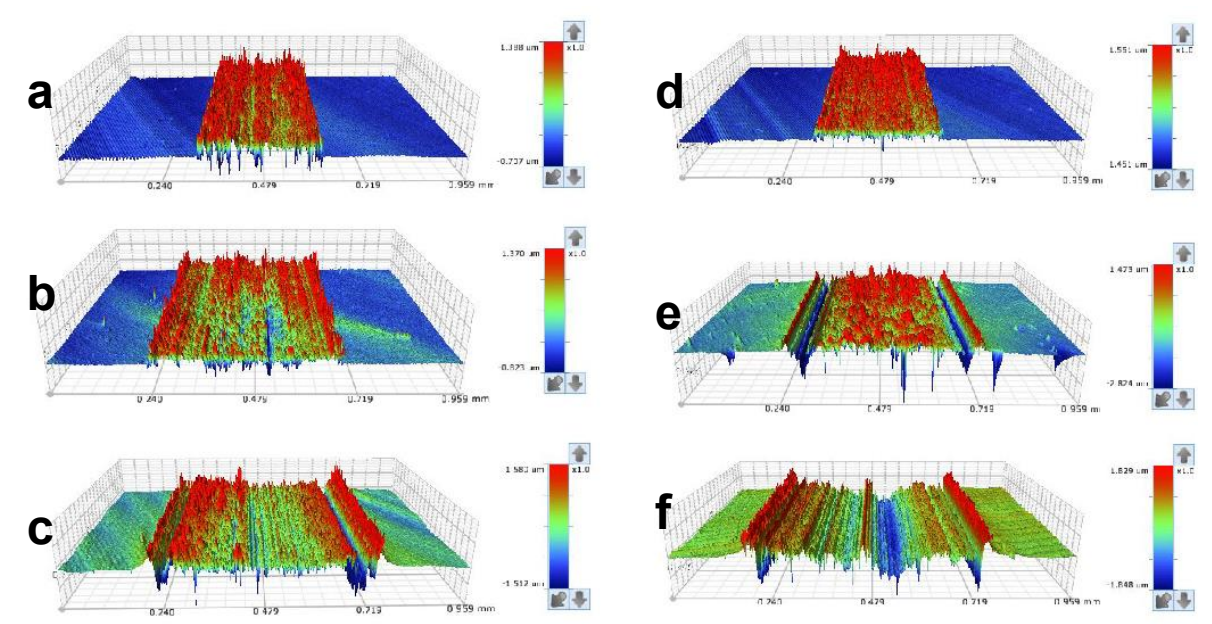

Figure S5. Three-dimensional morphology of wear scars of lower steel block lubricated with DIOS-cubic Ni nanoparticles (a, b, c) and DIOS-ellipsoidal Ni nanoparticle (d, e, f) at different loads under $20 \mathrm{mT}$ of magnetic field: (a, d) $100 \mathrm{~N},(\mathrm{~b}, \mathrm{e}) 300 \mathrm{~N}$, and (c, f) $500 \mathrm{~N}$ (frequency: $5 \mathrm{~Hz}$, amplitude: $5 \mathrm{~mm}$, additive concentration: $1 \%$ ). 

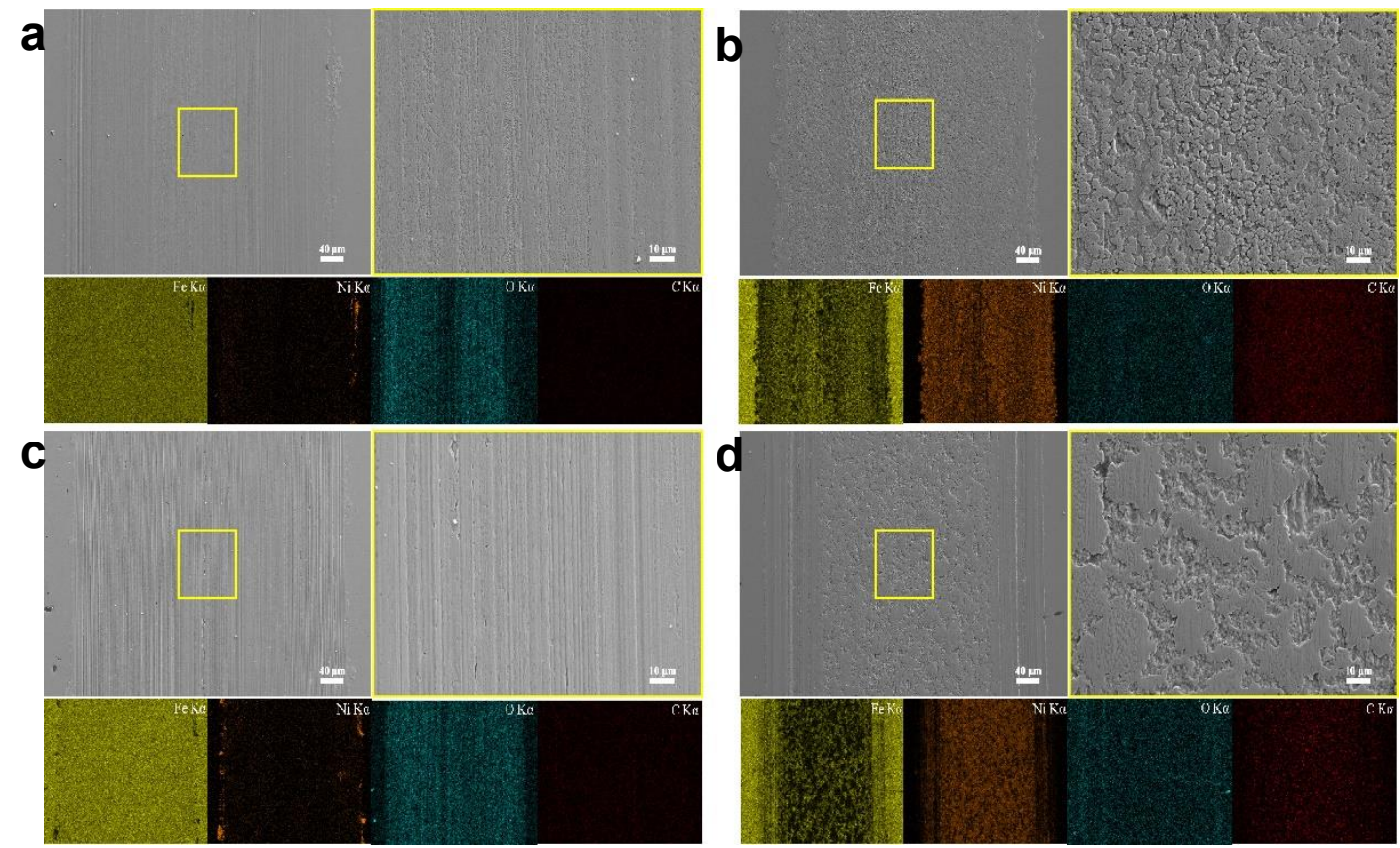

Figure S6. SEM images and EDS surface distribution of typical elements of the wear scar of lower steel block lubricated with DIOS-Ni nanoparticles at $300 \mathrm{~N}$ and $5 \mathrm{~Hz}$ : (a) DIOS $+1 \%$ cubic Ni at $0 \mathrm{mT}$, (b) DIOS $+1 \%$ cubic $\mathrm{Ni}$ at $20 \mathrm{mT}$, (c) DIOS $+1 \%$ ellipsoidal $\mathrm{Ni}$ at $0 \mathrm{mT}$, and (d) DIOS+1\% ellipsoidal $\mathrm{Ni}$ at $20 \mathrm{mT}$ (Load: $300 \mathrm{~N}$, frequency: $5 \mathrm{~Hz}$, amplitude: $5 \mathrm{~mm}$, additive concentration: $1 \%$ ). 

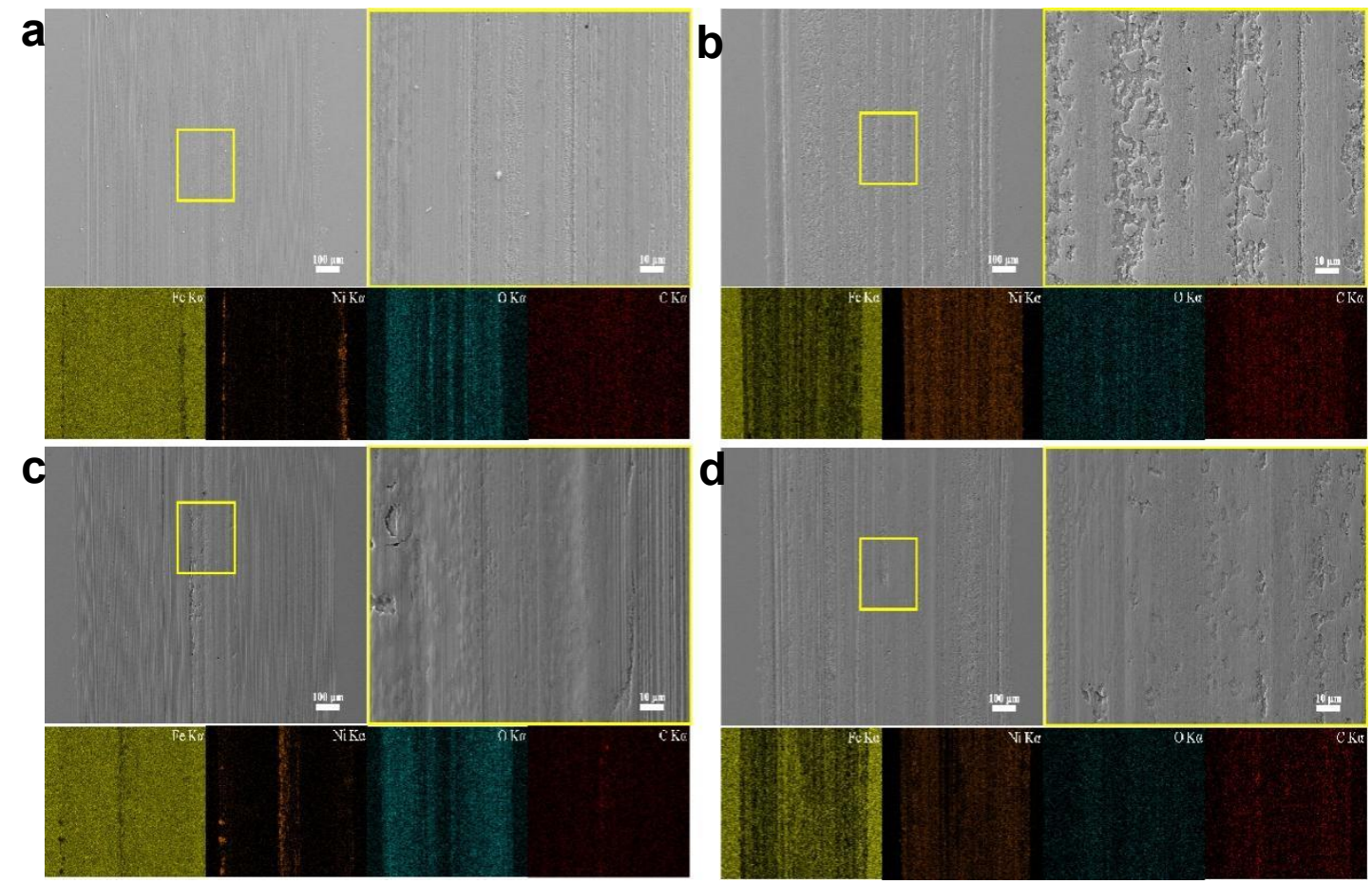

Figure S7. SEM images and EDS surface distribution of typical elements of the wear scar of lower steel block lubricated with DIOS-Ni nanoparticles at $500 \mathrm{~N}$ and $5 \mathrm{~Hz}$ : (a) DIOS $+1 \%$ cubic $\mathrm{Ni}$ at $0 \mathrm{mT}$, (b) DIOS $+1 \%$ cubic $\mathrm{Ni}$ at $20 \mathrm{mT}$, (c) DIOS $+1 \%$ ellipsoidal $\mathrm{Ni}$ at $0 \mathrm{mT}$, and (d) DIOS+1\% ellipsoidal Ni at $20 \mathrm{mT}$ (Load: $500 \mathrm{~N}$, frequency: $5 \mathrm{~Hz}$, amplitude: $5 \mathrm{~mm}$, additive concentration: $1 \%$ ). 

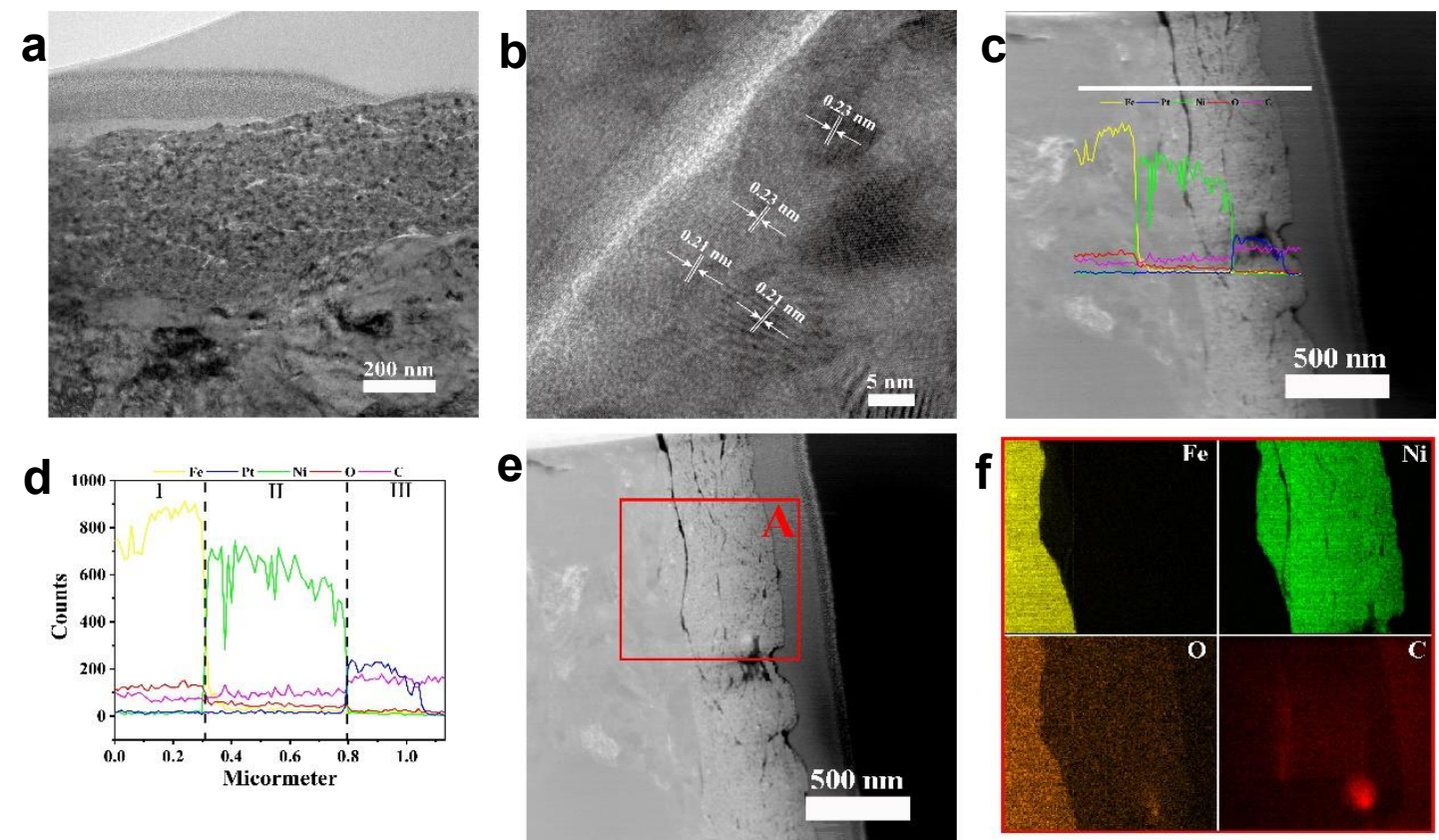

Figure S8. TEM images, EDS line scan images and EDS surface scan images of crosssection of wear scar of lower steel block lubricated with DIOS-ellipsoidal Ni nanoparticle (Load: $100 \mathrm{~N}$, frequency: $5 \mathrm{~Hz}$, amplitude: $5 \mathrm{~mm}$, magnetic field: $20 \mathrm{mT}$, additive concentration: $1 \%$ ). 

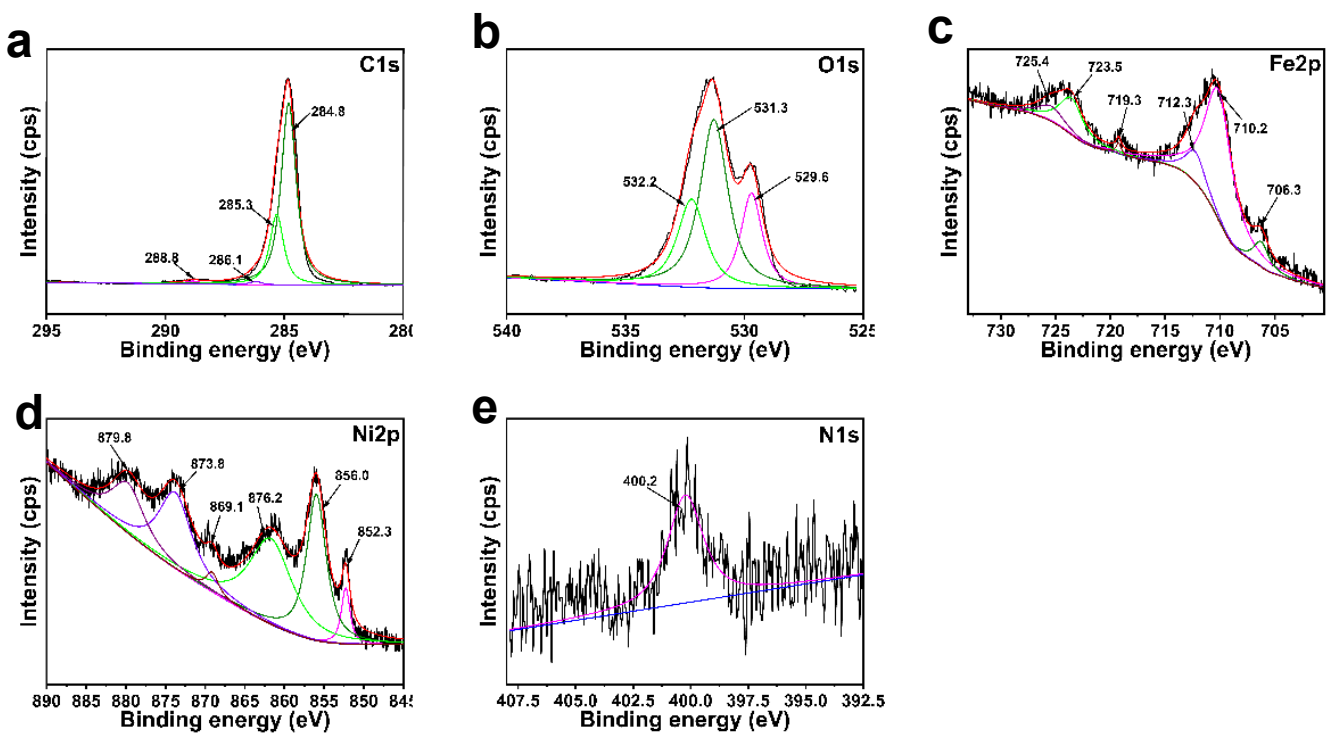

Figure S9. High-resolution XPS spectra of typical elements in wear scar of lower steel block lubricated with DIOS $+1 \%$ ellipsoidal Ni (Load: $100 \mathrm{~N}$, frequency: $5 \mathrm{~Hz}$, amplitude: $5 \mathrm{~mm}$, magnetic field: $20 \mathrm{mT}$ ). 